\title{
Not our cup of tea: the Tea Bag Index (Kueskamp et al. 2013) for assessing decomposition is problematic in most environments, due to macrofauna
}

\author{
Paul Eggleton ${ }^{1}$, Hannah Griffiths ${ }^{2}$, Louise Ashton ${ }^{3}$, Stephanie Law ${ }^{2}$, T.A. Evans ${ }^{4}$, and \\ Kate Parr $^{2}$ \\ ${ }^{1}$ Natural History Museum \\ ${ }^{2}$ University of Liverpool \\ ${ }^{3}$ University of Hong Kong \\ ${ }^{4}$ University of Western Australia
}

November 10, 2020

\begin{abstract}
1. The Tea Bag Index (Keuskamp et al. 2013) has been developed to allow worldwide comparable data on litter decomposition and has become an important part of several citizen science projects. This commentary shows that it is unsuitable for assessing total decomposition in most environments as it does not account for the role of macrofauna in the decomposition process. 2 . Tea bags were placed following the standardised protocol in old growth forest in Borneo (Sabah, East Malaysia). 3. The results shows that more than half of the bags were attacked by termites and they tended to remove the majority of the tea, rendering the Tea Bag Index incalculable. 4. The Tea Bag Index can measure the microbial component of decay, but will significantly underestimate total decay rates in most environments. This method will not give an accurate estimate of decomposition in most biomes.
\end{abstract}

\section{Introduction}

Decomposition is a vital part of ecosystem functioning, contributing fundamentally to nutrient cycling (Bazzaz 1998). It is recognised as one of the most important ecological processes (Krishna \& Mohan 2017), and so measuring it accurately is important; for this purpose it is necessary to find standardised methods, and the easier the method, the more widely it can be used.

The Tea Bag Index was proposed by (Keuskamp et al. 2013) as a standardised protocol to quantify microbial decomposition. It is a simple and attractive idea. Using just two different types of commercially available tea bags, bags are deployed for 90 days and, from their final weight, a measure of decomposition, known as the Tea Bag Index (TBI), is calculated. The method is intended for researchers and other interested people without a background in decomposition research, and is perfectly suited to citizen science projects (http://www.teatime4science.org/, www.spotteron.com/teabagindex). The method has now been widely used across numerous countries and has formed the basis for a various scientific publications (Ogden 2017; Becker \& Kuzyakov 2018).

The approach is ingenious. It uses two types of teabags: rooibos and green tea. The aim was to develop a method that will allow a single placement of teabags and enable the decay constant $k$ to be calculated for the slower decaying tea. The theory behind this is well established - there is, in every decaying piece of plant material, a slow ("refractory") and a fast ("labile") decomposing fraction which, under ideal conditions, will all decay (i.e. those under conditions of highest decomposition). Not all of the labile fraction of the rooibos 
tea will decay, slowed due toa larger recalcitrant fraction in the rooibos tea than in the green tea. The labile fraction has been estimated chemically, as 0.842 in green tea and 0.552 in rooibos tea (see Keuskamp et al. 2013). Under most natural conditions, not even all of the labile fraction of the green tea will have decomposed; a measure of this is $\mathrm{S}$, the stabilisation factor. $\mathrm{S}$ is calculated by assessing the proportion of the potential labile fraction that remains. Assuming that the rooibos tea shows a similar rate of loss of the labile fraction to the green tea, but that that fraction is a smaller proportion of the total, then proportional loss of the labile fraction per unit time of the rooibos tea can be calculated, and therefore $k$, the decomposition rate calculated. The measures $k$ and S are then used as the "Tea Bag Indices". Figure 3 in Keuskamp et al. (2013) shows these two estimates for sites across a range of habitats from rain forest to deserts with higher values in rain forest and lower values in desert.

Determing the Tea Bag Index relies on the important assumption that all agents of decomposition (i.e. microbe and invertebrates) are included in the assay. However, this may not be the case in all circumstances. In colder temperate and boreal regions fungal decay is by the far the most important factor in dead plant decay, but in hotter and wetter, more tropical, regions, a large portion of decomposition is carried out by macrofauna (especially termites; (Cornwell et al. 2009); (Handaet al. 2014; Griffiths et al. 2019). In temperate regions invertebrates such as earthworms, woodlice and millipedes also play an important role, albeit secondary to microbes (Wall et al. 2008; Smith et al. 2009).

Furthermore, termites need special consideration for two reasons. First, they are dominant decomposers in many habitats between $45^{\circ}$ north and south (Tuma, Eggleton \& Fayle In press).Second, termites can chew through many materials, including plastics (Lenz et al. 2012), and so may gain access to the tea leaves in the bags (i.e. chew the bags open - this is true for all types of litter bags). When this happens, the termites frequently consume all the contents of the tea bags; consequently the resulting data will have more zeros than those expected due to microbial decomposition alone. Under these conditions, it is impossible to calculate the real rate of ' $k$ ', as noted by Keuskamp et al. 2013 for samples left for 12 months . Additionally, the assumption that the recalcitrant fraction is essentially untouched is violated by termites (or other invertbrates that gain access) because they may remove a large proportion of tea, of both fractions. Therefore, in the presence of termites, removal of the tea from the bag will appear as the total decay of the tea by microbial agents, resulting in type I statistical errors. With these concerns in mind, here, we test the Tea Bag method to assess its performance in Bornean tropical rain forest (a hot and humid environment) with a high density of termites (Ashtonet al. 2019).

\section{Methods}

We conducted the experiment in the Maliau Basin Protected Area in a region of lowland old growth dipterocarp forest in Bornean Malaysia (the area is described in (Griffiths et al. 2018) and in (Ashtonet al. 2019). In order to test the method, we buried 60 green tea bags and 60 rooibos tea bags (of a known weight) at random within the forest at a depth of $8 \mathrm{~cm}$ as recommended by Keuskamp et al. (2013). We collected 20 of each type sacrifically at 7,14 and 60 days. We weighed each of the bags after collection and noted if they had been chewed opened by macrofauna. We left them out for 60 days rather than the recommended 90 days as our preliminary work showed that by 90 days most of the bags had holes in them and many were emptied. Keuskamp et al 2031 recommended reducing incubation time in "extreme sites... with extremely high k values (e.g. sites with high temperature and precipitation like site 13 in their study - which was rainforest in Panama).

In order to examine how effective the method is in tropical rain forest, we investigated the relationship between weight loss, the time that the bags were left out, and whether the bags had holes in them (i.e. opened by macrofauna). We expressed this as a \% mass loss per bag, which was logit transformed for analysis (following (Warton \& Hui 2011) so that we could use standard Gaussian linear models. Using R (R core team, 2013), we also tested the following full-interaction model, using thelm command:

$l g \sim$ days_gnd *hole_tbN * type_tbN

Where $\lg =$ logistic transformed mass-loss data; days_gnd = number of days the tea bags were left in the 
ground; hole_tbn $=$ the presence of holes in the tea bag; type_tbN $=$ the type of tea used $(G$ or $R)$.

\section{Results and Discussion}

After 60 days on the forest floor, we found that just over half of the bags of both tea types had been chewed through by macrofauna: 31 [52\%] of the rooibos tea and 30 bags [50\%] of the green tea. In our model the only significant terms were the type of tea (rooibos or green) and the interaction between the state of the bags (opened or unopened) and the tea type (see Table 1 for model outputs). There was no significant effect of time on the decomposition rate. This meant that generally green tea decomposed more rapidly than the rooibos tea. However, where the bags were opened, rooibos tea showed a greater response than in green tea, where the open and closed bags had very similar decay slopes (Table 1, Fig 1). This serves to illustrate that the major problem with the TBI in the tropics is with the rooibos tea bags, which were commonly attacked by macrofauna (probably predominantly termites) and the contents often completely removed. In our study, more than half of the rooibos tea bags were chewed open, and more than $10 \%$ were emptied. A similar proportion of green tea bags were chewed open, but none of them were emptied. It seems that macrofauna, at least in this context, prefer rooibos tea to green tea.

It is possible to estimate the two tea bag parameters in a subset of bags without holes (i.e. estimate from the undamaged bags, estimating microbial and mesofaunal decomposition only); the values here were $\mathrm{S}=$ 0.242 and $k=0.053$. This $k$ value from Bornean rainforst is higher than any values in the original teabag paper, including the tropical rain forest site in Panama $(\mathrm{S}=0.06, \mathrm{k}=0.0392$; Keuskamp et al. 2013 site 13, Table S1). This is, however, a measure of microbial decay only, as the macrofauna were specifically excluded. Keuskamp et al. 2013 could not calculate the standard deviation for their Panama site, 'due to overdispersion' of data. It is possible that holded teabags, empty of tea, may have contributed to this overdispersion.

However, it is difficult to assess projects that have used the TBI in the exact way it was recommended, as the method specifically excludes bags that have been chewed. Therefore they are self-selected for microbial and mesofauna decay and explicitly exlude any macrofauna decay.

If the aim is to quantify microbial decomposition it is therefore possible if only undamaged bags are used. However, sufficient bags need to be buried so that one can afford to ignore damaged ones. We suggest that given half of the bags were damaged at least twice as many are needed ( $>40$ in this case for every period, giving a total of 120). Furthermore, the incubation time (ie the time the tea bags are left out) should be reduced to 60 days, as by 90 days many of the teabags would be likely to be chewed by termites (note we recorded $50 \%$ ).

The TBI is a valuable idea but our results show it can only give an accurate idea of total decomposition rates in the few locations where invertebrate decomposition is negligible and there is overwhelmingly microbial decomposition (e.g. boreal areas). It works as a measure of microbial decomposition alone in all locations provided the bags are not damaged. Unfortunately, the TBI does not incorporate a measure for including the effects of larger decomposers.

We see no easy way to incorporate the contribution of macrofauna (particularly termites) to decomposition processes using the TBI, without also putting tea bags into macroinvertebrate litter bags made with mesh strong enough to withstand termite attack (such as stainless steel mesh or nylon; (Yates \& Grace 1999; Lenz et al. 2012). Other papers that use small and large mesh sizes to exclude or include macrofauna may suffer the same issue (eg (Handa et al. 2014)) if the small sized mesh material is not strong enough, then some samples may (are likely) to be attacked by termites.

This is because of the very high stochasticity that termites, in particular, contribute to the process. The problem becomes one of modelling two different simultaneous processes: one an exponential decay process and the other potentially a binomial process (either the bags are opened or they are not). There are other more costly methods, in both time and expertise, that produce robust total decomposition results and assess the contribution of all decomposer organisms, such as using leaf litter and wood decomposition assays and mesh to include/exclude invertebrates (e.g. (Smith et al. 2009; Davies et al.2013). However, these methods 
are not in the spirit of the TBI (see Fig 1), which was designed to produce a quick, simple estimate of two meaningful parameters.

In summary, the TBI can work well to assess the microbial contribution to decomposition where no (or few) bags are opened by macrofauna, such as in boreal locations, but the method risks seriously underestimating total (microbial + macrofauna) decomposition in most habitats. (Wallet al. 2008) present a global map showing the areas where macrofauna (not always termites) are important in dead plant decomposition processes, althoughthis figure significantly downplays the effect of macrofauna (especially termites) in savannas, grasslands and other drier areas (Bignell \& Eggleton 2000). Wall et al. 2008 used moth balls (active ingredient naphthalene) to exclude invertebrates. As with any insecticidal active ingredient, it has variable effects on different species of insect...

Termites appear to manufacture naphthalene (possibly secreted by themselves, but more likely via microbes(Chen et al. 1998)). So the treatement would have a reduced effect on termites.

While Keuskamp et al. (2013) do specifically state that macrofauna are excluded they make no other comment about the errors that this might introduce. We believe that any method that ignores the contribution of macrofauna to plant decomposition cannot be recommended as a global method. It may still be useful for estimating purely-microbial effects but it cannot yield measures that include all decay agents.

Data accessibility: the data for this paper has been deposited in DRYAD, doi: TBA

Table 1

\begin{tabular}{llll}
\hline Variable & Estimate & Std. Error & t-value \\
\hline intercept & -0.703 & 0.123 & $-5.696^{* * *}$ \\
Number of days & 0.006 & 0.004 & 1.61 n.s. \\
Presence of holes & 0.042 & 0.186 & 0.83 n.s. \\
Type of tea & -0.774 & 0.175 & $-4.427^{* * *}$ \\
Days*holes & 0.000 & 0.006 & 0.983 n.s. \\
Days*type & 0.006 & 0.006 & 0.315 n.s. \\
Type*holes & 0.661 & 0.264 & $2.505^{*}$ \\
Days*holes*type & -0.009 & 0.008 & 0.2625 n.s. \\
\hline
\end{tabular}

Table 1. Result of fully interactive linear model testing the effect our focal variables on the mass lost from rooibos and green tea bags. Asterix indicate significant effects ${ }^{*} P<0.05,{ }^{* * *} \mathrm{P}<0.001$ and n.s. non-significant. 


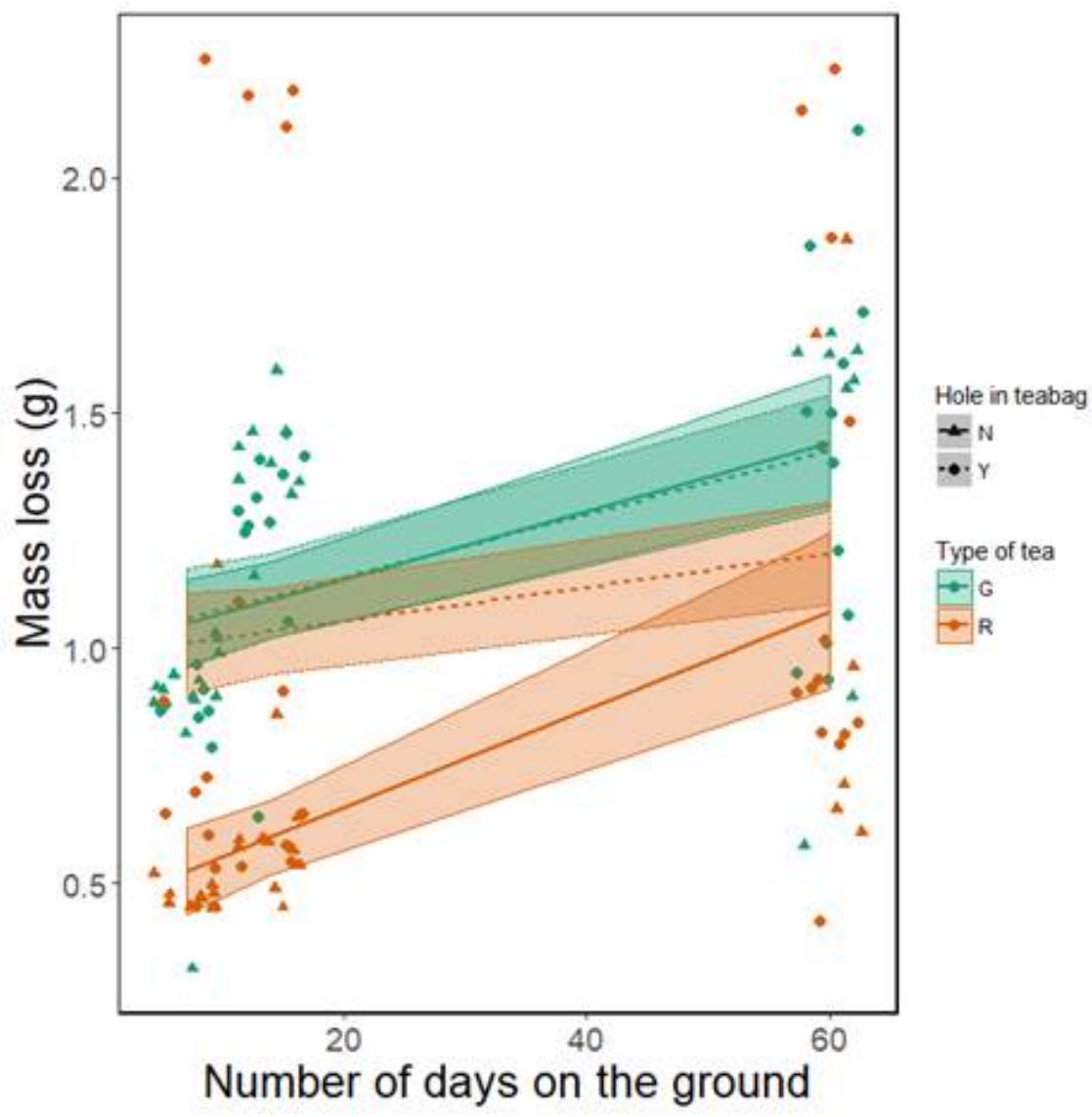

Figure 1. The significant effect of the interaction between holes in teabags (triangles are teabags without holes, circles are those with holes) and tea type (green tea $=$ green points, rooibos $=$ orange points). Lines display fitted values from linear model outputs. Green lines are green tea, organe lines are rooibos, dashed lines are teabags with holes, and solid lines are those without. The ribbons show standard errors around model fitted values. There is clear difference in the response to having holes of the two types of tea: The green tea shows little difference between opened (ie chewed open) and unopened (ie intact) bags; the rooibos clearly shows more decomposition when the bags have holes.

\section{References}

Ashton, L.A., Griffiths, H.M., Parr, C.L., Evans, T.A., Didham, R.K., Hasan, F., Teh, Y.A., Tin, H.S., Vairappan, C.S. \& Eggleton, P. (2019) Termites mitigate the effects of drought in tropical rainforest.Science, 363, 174 .

Bazzaz, F. (1998) Tropical forests in a future climate: Changes in biological diversity and impact on the global carbon cycle.Climatic Change, 39, 317-336.

Becker, J.N. \& Kuzyakov, Y. (2018) Teatime on Mount Kilimanjaro: Assessing climate and land-use effects on litter decomposition and stabilization using the Tea Bag Index. Land Degradation 83 Development, 29, 2321-2329.

Bignell, D. \& Eggleton, P. (2000) Termites in Ecosystems. DE Bignell - Termites: evolution, sociality, symbioses, ecology, 2000. Kluwer Academic Publishers.

Chen, J., Henderson, G., Grimm, C.C., Lloyd, S.W. \& Laine, R.A. (1998) Termites fumigate their nests with 
naphthalene. Nature,392, 558-559.

Cornwell, W.K., Cornelissen, J.H.C., Allison, S.D., Bauhus, J., Eggleton, P., Preston, C.M., Scarff, F., Weedon, J.T., Wirth, C. \& Zanne, A.E. (2009) Plant traits and wood fates across the globe: rotted, burned, or consumed? Global Change Biology, 15,2431-2449.

Davies, A.B., Van Rensburg, B.J., Eggleton, P. \& Parr, C.L. (2013) Interactive Effects of Fire, Rainfall, and Litter Quality on Decomposition in Savannas: Frequent Fire Leads to Contrasting Effects.Ecosystems, 16, 866-880.

Griffiths, H., Ashton, L., Walker, A., Hasan, F., Evans, T., Eggleton, P. \& Parr, C. (2018) Ants are the major agents of resource removal from tropical rainforests. Journal of Animal Ecology, 87,293-300.

Griffiths, H.M., Ashton, L.A., Evans, T.A., Parr, C.L. \& Eggleton, P. (2019) Termites can decompose more than half of deadwood in tropical rainforest. Current Biology, 29, R118-R119.

Handa, I.T., Aerts, R., Berendse, F., Berg, M.P., Bruder, A., Butenschoen, O., Chauvet, E., Gessner, M.O., Jabiol, J., Makkonen, M., McKie, B.G., Malmqvist, B., Peeters, E.T.H.M., Scheu, S., Schmid, B., van Ruijven, J., Vos, V.C.A. \& Hättenschwiler, S. (2014) Consequences of biodiversity loss for litter decomposition across biomes. Nature, 509, 218-221.

Keuskamp, J.A., Dingemans, B.J.J., Lehtinen, T., Sarneel, J.M. \& Hefting, M.M. (2013) Tea Bag Index: a novel approach to collect uniform decomposition data across ecosystems. Methods in Ecology and Evolution, 4, 1070-1075.

Krishna, M.P. \& Mohan, M. (2017) Litter decomposition in forest ecosystems: a review. Energy, Ecology and Environment,2, 236-249.

Lenz, M., Creffield, J.W., Evans, T.A., Kard, B., Vongkaluang, C., Sornnuwat, Y., Lee, C.-Y., Yoshimura, T. \& Tsunoda, K. (2012) Resistance of polyamide and polyethylene cable sheathings to termites in Australia, Thailand, USA, Malaysia and Japan: A comparison of four field assessment methods. International Biodeterioration \&3 Biodegradation, 66, 53-62.

Ogden, L.E. (2017) Brewing Big Data: The Tea-Bag Index.BioScience, 67, 680-680.

Smith, J., Potts, S.G., Woodcock, B.A. \& Eggleton, P. (2009) The impact of two arable field margin management schemes on litter decomposition. Applied Soil Ecology, 41, 90-97.

Tuma, J., Eggleton, P. \& Fayle, T. (In press) Ant-termite interaction: an important but under-explored ecological linkage. Biological Reviews .

Wall, D.H., Bradford, M.A., St. John, M.G., Trofymow, J.A., Behan-Pelletier, V., Bignell, D.E., Dangerfield, J.M., Parton, W.J., Rusek, J., Voigt, W., Wolters, V., Gardel, H.Z., Ayuke, F.O., Bashford, R., Beljakova, O.I., Bohlen, P.J., Brauman, A., Flemming, S., Henschel, J.R., Johnson, D.L., Jones, T.H., Kovarova, M., Kranabetter, J.M., Kutny, L.E.S., Lin, K.-C., Maryati, M., Masse, D., Pokarzhevskii, A., Rahman, H., SabarÁ, M.G., Salamon, J.-A., Swift, M.J., Varela, A., Vasconcelos, H.L., White, D.O.N. \& Zou, X. (2008) Global decomposition experiment shows soil animal impacts on decomposition are climate-dependent. Global Change Biology, 14, 2661-2677.

Warton, D.I. \& Hui, F.K.C. (2011) The arcsine is asinine: the analysis of proportions in ecology. Ecology, 92, 3-10.

Yates, R. \& Grace, J. (1999) TERMITE RESISTANT CONSTRUCTION AND BUILDING MATERIALS. Proceedings of the 3rd International Conference on Urban Pests, 3, 399-406. 